\title{
Intervention of Diet in Psoriasis: A Review
}

\section{Abdul Haque Bamer ${ }^{1 *}$, Abdul Samad ${ }^{2}$, Prasad Govindrao Jamkhande ${ }^{3}$, Abid Shaikh ${ }^{1}$, Nadeem Shaikh ${ }^{4}$, Sumayya Fatema ${ }^{1}$ and Quadri Mohammad Shoeb ${ }^{1}$}

${ }^{1}$ Department of Pharmacology, Durgamata institute of Pharmacy, Dharmapuri, Maharashtra, India

${ }^{2}$ Department of Clinical Pharmacy, Poona College of Pharmacy, Bharati Vidyapeeth

(Deemed to be) University, Maharashtra, India

${ }^{3}$ Center for the Research in Pharmaceutical science, Sharda Bhavan Education

Society's Nanded college of Pharmacy, Maharashtra, India

${ }^{4}$ School of Pharmacy, S.R.T.M. University, Maharashtra, India

*Corresponding Author: Abdul Haque Bamer, Department of Pharmacology,

Durgamata Institute of Pharmacy, Dharmapuri, Maharashtra, India.
Received: August 14, 2021

Published: September 23, 2021

(C) All rights are reserved by Abdul Haque

Bamer., et al.

\begin{abstract}
Psoriasis is a chronic disfiguring, Relapsing, inflammatory and proliferative immune mediated skin disease. The correct aetiology is unclear but a mix of genetic, stress and environmental factors is considered as the cause. The disease is associated with anxiety, depression, crohn's disease cardiovascular disease, obesity and metabolic syndrome. The current research on diet in psoriasis as reason and cure has gained attention with numerous studies correlating it with aetiology and pathogenesis of the disease. In this review, diet considered an important role in psoriasis patients. The main aim of review to discuss the different dietary interventions proposed for the management of psoriasis, the data concerning the same and controversies surrounding them. Low caloric diet has shown to increased severity of psoriasis of skin lesion. Antibodies to gluten may be seen in or else asymptomatic patients of psoriasis and these may advantage from a gluten free diet. Omega-3 fatty acids have revealed a strong evidence to be beneficial in several trials. But, the dose and route of administration is yet to be standardized. The alcohol addiction of patients with psoriasis has led to its worsening. Amongst the vitamins, vitamin D shows the maximum evidence of benefit, while the role of folate and vitamin B12 needs to be explored further. Similar is the case for zinc and selenium. There is evidence of worsen of psoriasis with foods such as eggs, red meat and dairy products and those rich in taurine, but the evidence is too inadequate to advise decrease in intake of these items. Dermatologists must be conscious of this management to help their patients put together the best option for dietary modification.
\end{abstract}

Keywords: Psoriasis; Diet; Dietary Intolerance; Vitamins; Antioxidants

\section{Abbreviations}

BMI: Body Mass Index; PASI: Psoriasis Area Severity Index; DLQI: Dermatological Life Quality Index; EPA: Eicosapentaenoic;
DHA: Docosahexanoic; NAPSI: Nail Psoriasis Severity Index; Hcy: Homocysteine; HHcy: Hyperhomocysteinaemia; MDA: Malondialdehyde; TAC: Total Antioxidant Capacity; AA: Arachidonic Acid 


\section{Introduction}

Psoriasis is a common chronic, inflammatory, skin disease that results from a malfunction of immune system. The name psoriasis is from the Greek language, meaning "roughly itching condition" (psora: "itch", sis: "action") Psoriasis is a non-infectious, dry, inflammatory and ugly skin disorder, which can involve entire system of person [1]. The skin becomes irritated, and skin cell proliferation accelerates. Typical symptoms include red spots on the skin, inflamed skin, silvery scales, and itching. Plaques can affect any area of the body with different intensity, most notably the elbow, scalp, knees, skin, and joints. Plaques can appear as a single spot or cover the entire body's surface. Skin gradually accumulates at the areas, resulting in a white silvery appearance [2]. Pathophysiology behind the development of psoriasis is T-cell activation, which migrates from lymph nodes and the systemic circulation to the skin and triggers the release of cytokines, which drive cutaneous inflammation and epidermal hyperproliferation, resulting in erythematous, elevated plaques with overlaying scale, is the pathophysiology underlying the development of psoriasis [3]. Psoriasis affects between $1 \%$ and $3 \%$ of the population. The hyperproliferation of keratinocytes caused by an active immune system distinguishes psoriasis. Psoriasis can emerge at any age, although it is most common between the ages of 15 and 35. Some people inherit genes that make them more prone to psoriasis, however this does not mean that they will develop it. One-third of persons with psoriasis have had at minimum one affected family member. Smoking, drinking, sun exposure, skin damage, and stress are all thought to play a role in the development and severity of psoriasis. Specific variables, such as increasing body mass index and weight gain, have been linked to a higher prevalence of psoriasis and are risk factors for the disease. Because the severity and frequency of psoriasis vary, individuals can often suffer a serious negative impact on their quality of life, with higher incidences of anxiety and depression [4]. The role of nutrition and diet in management of psoriasis has been studied since long. Their role has now been established in the etiopathogenesis of psoriasis by several authors [5].

The association of metabolic syndrome and psoriasis has increased attention in either field. In clinical settings, patients commonly seek information about dietary interventions. As a result, in order to better advice patients, the treating dermatologist must be up to date on the most recent evidence on dietary interventions. The purpose of this review is to highlight the positive and negative impacts of various dietary interventions in the treatment of psoriasis. The strength of the evidence as well as the issues surrounding these interventions will be discussed.

\section{Hypocaloric diet}

Diet plays a significant role in decreasing the severity of psoriasis. Numerous studies have linked an elevated BMI to a greater incidence and severity of psoriasis [6]. Obese patients are shown to be twice as likely as healthy subjects to get psoriasis. They found that increasing BMI by one unit increased the probability of psoriasis development by $9 \%$ and elevated the Psoriasis Area Severity Index [PASI] by 7\%. [7]. It is stated that patients with moderate - to - severe psoriasis with a BMI more than 30 , who were given a reduced energy diet and cyclosporine showed a 75\% in PASI [PASI 75] compared to the group on cyclosporine alone [8]. When the incidence of psoriasis was found to have decreased during Second World War the observation that psoriasis responds to a low energy diet was made [9]. In the randomized controlled trial found that the group of 30 patients on a low energy diet showed a significantly greater reduction in weight than the control group on normal diet. The test group even had a larger decrease in PASI and DLQI [10]. Another study indicated that an investigator-blinded, randomized controlled trial revealed that psoriasis patients stabilized on methotrexate and on a reduced calorie diet took a longer time for their illness to relapse [11].

\section{Vegetarian diet}

Diet has an important role in the treatment of a variety of skin disorders. Vegetarian diets have been studied for their ability to alleviate the symptoms of chronic inflammatory diseases. Fresh green leafy vegetables and fruits were linked to a decreased incidence of psoriasis. This will not cure psoriasis, but it may significantly reduce its severity. A vegetarian diet can be nutritionally adequate and have potential health advantages, even for those with psoriasis, if it is carefully planned and organized [12]. Psoriasis was shown to be inversely related to the consumption of vegetables such as carrots, tomatoes, and fresh fruits. A vegetarian diet is low in arachidonic acid, which reduces the production of inflammatory eicosanoids. In one research, the addition of an omega- 3 fatty acidrich diet to a vegetarian diet alternated with periods of hypocaloric diet was shown to be helpful [13]. The advantages of such a diet have been attributed to lower levels of arachidonic acid and its proinflammatory metabolites [14]. 


\section{Gluten free diet}

The identical Th1 cytokine profile found in both illnesses has been linked to the association between psoriasis and celiac disease. The release of IL1 and IL8 from rapidly proliferating keratinocytes results in the production of this Th1 cytokine profile [15]. Michaelsson and colleagues documented 33 AGA-positive psoriasis patients who were placed on a gluten-free diet for three months. PASI scores improved significantly in 73 percent of these individuals (from an average of 9.0 to 5.5 in patients with original PASI greater than 5). Furthermore, when these individuals returned to their previous diets, their PASI scores recovered to prediet levels [16]. Further research has revealed not only a reduction in tTG expression in skin lesions, but also a reduction in proliferating epithelial cells (Ki67+ cells) in the papillary dermis in patients on a gluten-free diet who are not receiving any other psoriasis treatment. Patients who did not have increased AGAs and followed a gluten-free diet for the same three months exhibited no difference in epidermal histology. Tissue transglutaminase levels are shown to be higher in psoriatic skin and decrease once a gluten-free diet is implemented [17]. Some researchers have questioned the favorable link between the two diseases. Thus, a gluten-free diet may be beneficial in psoriasis patients with celiac disease antibodies, but further research is needed to reach a conclusion [15].

\section{0mega-3 fatty acids}

Cold water fish oils high in omega-3 polyunsaturated fatty acids, eicosapentaenoic acid (EPA), and docosahexanoic acid (DHA) have been studied for use in the treatment of psoriasis. By competition, omega-3 fatty acid consumption reduces the synthesis of arachidonic acid-derived proinflammatory mediators and stimulates the amount of metabolites that are less inflammatory in nature than the arachidonic acid metabolite. Their effects might also be attributed to changes in intracellular signaling pathways, antioxidant activity, and transcription factor activity modulation [18]. Several studies have been done to assess the effect of omega 3 fatty acids. This research looked at the effectiveness of an omega- 3 fatty acidrich dietary supplement in individuals with mild to severe plaque psoriasis. Thirty patients were enrolled, with 15 of them receiving tacalcitol topical therapy as the control group. The remaining 15 patients were given topical tacalcitol and two Oravex ${ }^{\circledR}$ tablets daily. Over an 8-week period, three visits were conducted: the baseline, intermediate (week 4), and final (week 8).The main efficacy endpoints were the Psoriasis Area and Severity Index (PASI), Nail Psoriasis Severity Index (NAPSI) and Dermatological Life Quality Index (DLQI).Between the baseline and end visits, there was a clear and substantial improvement in all effectiveness endpoints in both groups. This improvement was substantially larger in the group treated with Oravex ${ }^{\circledR}$ in addition to the control group. Supplemental treatment with omega-3 fatty acids complements topical treatment in psoriasis and contributes significantly to lowering PASI and NAPSI and improving DLQI, as well as lowering scalp lesion and pruritus, erythema, scaling, and infiltration of the treated areas [19]. Mayser., et al. and Grimminger., et al. demonstrated the superiority of omega 3 fatty acids over omega- 6 fatty acids in decreasing the severity of the disease in chronic plaque and guttate psoriasis respectively. Several trials conducted double-blind, randomized, controlled studies comparing the effect of intravenous omega-3 fatty acids (Omegaven) to omega- 6 fatty acids (Lipoven) for the treatment of psoriasis. In the Mayser., et al. study, 75 subjects with chronic plaque psoriasis subjects were randomized to a 14-day treatment with either intravenous omega-3 or omega-6. PASI scores decreased by $11.2 \pm 9.8$ in the omega- 3 group versus $7.5 \pm 8.8$ in the omega -6 group ( $\mathrm{p}=0.048$ ), with significantly better improvement for the omega-3 group in erythema, scale, and induration. In Grimminger., et al. 20 subjects with acute guttate psoriasis received either intravenous omega- 3 or omega- 6 for 10 days. When compared to the omega- 6 group, the omega-3 group improved more in terms of erythema, scale, and induration. However, other studies have found that omega-3 fatty acids had no additional effect in psoriasis. This contains two randomised, double-blind, placebo-controlled trials, which weakens the case for omega-3 fatty acids. As a result, fish oils are recommended for psoriasis patients. The amount of omega-3 fatty acids required to reach a threshold level in epidermal phospholipids, which may block arachidonic acid-derived eicosanoids, is most likely high. More research is needed to determine an acceptable dose guideline for the same [20,21].

\section{Vitamins}

\section{Vitamin A}

Retinoids, which are vitamin A compounds, have been used topically and orally for decades. The initial retinoid mechanism was antiproliferative effects and epithelial differentiation promotion. Both efficiently inhibit keratinocyte hyperproliferation and modify the abnormal differentiation found in psoriasis. Serum levels have 
been observed to be lower in patients with various morphological forms of psoriasis, as well as in inactive disease [22]. Vitamin A's antipsoriatic action is limited, as larger dosages required for clearance may cause systemic toxicity. This prohibited any meaningful experiments testing vitamin A supplementation in psoriasis from taking place. However, vitamin A derivatives known as retinoids have been firmly established as a key armamentarium in the treatment of psoriasis [23].

\section{Vitamin B9 (Folic acid)}

Patients suffering from psoriasis may be deficient in folic acid. This is due to reduced intestinal absorption caused by inflammation, increased use by keratinocytes, and higher homocysteine levels, which is an independent risk factor for cardiovascular disease [24]. According to a case control study, obese psoriatic patients had low folate levels and high homocysteine levels. Because psoriasis is linked with an elevated risk of cardiovascular morbidity and mortality, and homocysteine is an independent risk factor for cardiovascular disease, it may seem reasonable that reducing this risk factor would have a positive impact on cardiovascular mortality and morbidity. The potential advantage of folate supplementation appears to be logical. Folate has long been utilised in the treatment of psoriasis, psoriatic arthritis, and rheumatoid arthritis in conjunction with methotrexate. It is beneficial in decreasing gastrointestinal side effects and abnormal liver function tests in this context. Methotrexate was found to lower the incidence of vascular disease in a retrospective cohort study of over 7000 psoriasis patients, and this reduction was further increased when folic acid was added. More research is needed before folate is recommended as a supplement for psoriasis patients with heart disease [25].

\section{Vitamin B12}

Deficiency of vitamin B12 may also contribute to the increase of Hcy in psoriasis [26]. Recent research has shown that psoriasis patients had considerably higher blood homocysteine (Hcy) levels and a greater incidence of hyperhomocysteinaemia (HHcy). Folic acid and vitamin B12 deficiency can be a cause of HHcy in psoriasis. Hcy may contribute to the pathogenesis of psoriasis by activating Th1 and Th17 cells, as well as neutrophils, while inhibiting regulatory T cells. Furthermore, Hcy can promote the immunoinflammatory process in psoriasis by increasing the production of pro-inflammatory cytokines. Hcy may activate nuclear factor kappa $\mathrm{B}$, which is important in the immunopathogenesis of psoriasis. The oxidative stress status in psoriasis and the impact of HHcy may be linked. Hydrogen sulphide (H2S) may have a protective role in the pathogenesis of psoriasis, and HHcy may induce H2S deficit in psoriasis. Because the function of Hcy in the aetiology of psoriasis is most likely established, Hcy might be a potential therapeutic target for psoriasis treatment. The use of systemic folinate calcium, a folic acid derivative, and topical vitamin B12 has been shown to be helpful in the treatment of psoriasis. More study is needed to determine the potential role of vitamin B12 in the treatment of early psoriasis [27].

\section{Vitamin D}

Psoriasis lesions are characterized by hyperproliferation of epidermal keratinocytes, which is accompanied by an inflammatory cellular infiltration in both the dermis and the epidermis. Sunlight activity on the epidermis is the natural source of vitamin D production. Vitamin D has become an essential local therapeutic option in the treatment of psoriasis due to its function in keratinocyte proliferation and maturation. It also possesses immunomodulatory properties in psoriasis, acting directly on T cells and antigenpresenting cells. According to studies, psoriasis patients have low serum vitamin D levels, and the severity of psoriasis is inversely related to serum vitamin D levels [28]. Several open trials of vitamin D supplementation in psoriasis have been performed, with the majority of patients responding well. Perez., et al. conducted the largest of these trials on 85 patients. Calcitriol supplementation provided some benefit to $88 \%$ of patients, with a fourth of all patients exhibiting full eradication of lesions and another third showing moderate improvement [29]. More research is needed to find the impact of oral vitamin D in psoriasis. Still, owing to the public health problem of vitamin $\mathrm{D}$, its supplementation may be recommended in patients not on topical vitamin D analogues [30].

\section{Antioxidants}

Selenium

Selenium has antioxidative, antiproliferative, immunomodulatory, and DNA synthesis properties, making it a possible therapy for psoriasis. According to research, psoriasis patients are low in selenium, and a lack of this essential micronutrient correlates with disease severity [31]. Madhulatha M and Vijayabhaskar M investigated the role of oxidative stress in the onset and progression of psoriasis, as well as the therapeutic effect of antioxidant therapy in psoriatic patients in addition to conventional treatment. Thirtythree patients of age group 20-60 years with chronic plaque psoriasis. The research included patients with disease duration varying from 6 months to 2 years. The psoriasis area severity index was used to determine the severity of the disease (PASI). Along with 
standard treatment, antioxidant therapy was administered for 8 weeks. Before and after the treatment, malondialdehyde (MDA) and total antioxidant capacity were measured and compared. After 8 weeks of antioxidant therapy, there was a significant decrease in mean serum MDA levels and an increase in total antioxidant capacity (TAC). The findings revealed that combining antioxidant therapy with conventional psoriasis treatment is more effective in the treatment of psoriatic cases [32]. However, whether combined with narrow band UVB radiation or topical therapy, selenium supplementation was not found to be superior [31].

Zinc

Combination of copper, zinc is a powerful antioxidant and is important for maintenance of normal immune responses. Estabraq ARK Alwasitiet., et al. investigated the relationship between oxidative stress parameters and trace elements such as zinc (zn) and copper (cu) in the pathogenesis of psoriasis disease. The research included 50 psoriasis patients. The study included 32 patients with localised psoriasis and 18 individuals with generalised psoriasis, as well as $\mathbf{5 0}$ healthy controls. It is suggested that lipid peroxidation of keratinocyte cellular membranes by free radicals and a reduction in antioxidant may be associated with the pathogenesis of psoriasis lesion. Furthermore, an enhanced diet or supplement of vitamin A, E, and zinc was found to be beneficial in the treatment of psoriasis. Plaques of psoriasis have been linked to zinc deficiency in some cases. In clinical trials, however, zinc supplementation failed to show any benefit in psoriasis lesions. The significance of zinc as an antioxidant in psoriasis has to be studied further, as it is a highly safe mineral when taken on a regular basis [33].

\section{Foods that worsen psoriasis}

The disease is exacerbating by numerous factors and tends to worsen with time. A variety of factors that lead to the start of psoriasis are discussed below.

\section{High glycemic foods}

Elevated glycemic foods increase insulin levels and have a greater prevalence of psoriasis than the general population. Foods with a high glycemic index induce insulin resistance and the activation of pro-inflammatory cytokine genes, resulting in Theoretical risk of psoriasis triggering or worsening [34]. Boencke., et al. proposed a positive correlation between PASI score and insulin levels. Thus, low glycemic index foods may become part of the general manage- ment of psoriatic patients because they minimize the risk of significant cardiovascular risk [13].

\section{Alcohol}

Extensive data has linked the quantity of alcohol consumed and the kind of beverage consumed to the onset and exacerbation of psoriasis. Despite the fact that the hypothesis that alcohol consumption is associated with an increased risk of psoriasis onset and worsening is not credible, studies have shown that alcohol can directly and indirectly increase the production of pro-inflammatory cytokines from many cell types, resulting in persistent systemic inflammation and lymphocyte proliferation. Furthermore, alcohol and acetone have been shown to directly stimulate keratinocyte proliferation and increase the mRNA levels of genes associated with proliferating keratinocytes, such as a5 integrin, cyclin D1, and keratinocyte growth factor receptor [35]. However, there is contradictory data as to whether higher alcohol intake in psoriasis patients is a role in pathogenesis or whether having a chronic disorder such as psoriasis leads to increased alcohol intake. One study of 144 psoriasis patients, for example, found that alcohol intake in the previous 12 months was associated to the onset of psoriasis. According to the study, psoriasis may lead to long-term alcohol abuse, and alcohol consumption may worsen psoriasis [36]. Nonetheless, alcohol consumption was found to be higher among psoriasis patients than in the general population. Although the link between psoriasis and alcohol consumption is complicated and multifactorial, alcohol abuse is associated with increased psoriasis severity and decreased treatment effectiveness [37].

\section{Smoking or tobacco consumption}

Because smoking has significant extra-pulmonary toxicity, it is an important lifestyle factor that influences human health and can impact the progression of psoriasis. Smoking, which has been shown to accelerate the development of comorbidities associated with psoriasis such as atherosclerotic heart disease and metabolic syndrome, is an independent risk factor for psoriasis [38]. Psoriasis is a $\mathrm{T}$ cell immune-mediated disease, and nicotine affects a variety of immunological processes, including innate and adaptive immune responses. Furthermore, nicotine intake improves the production of IL-12 and several other pro-inflammatory cytokines implicated in diseases, such as tumour necrosis factor [39]. Tobacco contains nicotine, which promotes dysregulated production of vascular endothelial growth factor, an essential element in angio- 
genesis. Because psoriasis is characterised by an increase in angiogenesis, this might explain a portion of the link between smoking and psoriasis. Long-term smoke exposure has also been linked to the development of psoriasis due to the fact that smoking causes oxidative stress and free radical damage. The increased presence of free radicals in the human body has the potential to trigger a cascade of systemic effects, including the development of psoriasis (40).

\section{Infection}

Toxins that activate T cells, such as bacterial toxins, lead to development of cutaneous lymphocyte antigen, which causes psoriatic lesions. Acute guttate psoriasis is closely linked to a previous or concurrent streptococcal infection, particularly of the throat. Streptococcal infection appears to be significant in persistent plaque psoriasis, and treatment with rifampicin and penicillin may lead in skin lesions clearing [41].

\section{Eggs, red meat and dairy products}

The severity of psoriasis has been linked to a diet high in red meat. Arachidonic acid, a polyunsaturated fatty acid found in red meat, dairy, and eggs, is a type of polyunsaturated fatty acid. According to study, arachidonic acid byproducts may have a role in the development of psoriatic lesions [42].

\section{Taurine rich diet}

The amino acid Taurine was considered to be involved in the pathogenesis of psoriasis in early studies, however a number of studies failed to confirm the assumption that taurine in any quantity can aggravate or improve the medical course of psoriasis. Increased consumption of taurine, an amino acid, in the diet was associated to psoriasis exacerbation and accompanying pruritus, and taurine's function in the pathogenesis of psoriasis was proposed. In another study, 15 patients with mild to severe psoriasis were given a low taurine diet, which ultimately resulted in total healing of 9 psoriatic patients and partial cure in the case of the remaining patient during a 3-month period. However, excess taurine did not produce exacerbation in patients in another trial [43].

\section{Obesity}

Several study reports suggested that obesity is the disease's causative agent, while others indicated that psoriasis causes obesity. It has been related to a variety of comorbidities such as car- diovascular disease, psoriatic arthritis, obesity, and metabolic syndrome. Obesity may be a risk factor in the development of psoriasis, exacerbate existing psoriasis, and weight loss may worsen the severity of psoriasis in overweight patients. Excess body weight can interfere with psoriasis treatment and increases the cardiovascular risk profile in these patients, emphasizing the necessity of appropriate weight-control programs [35].

\section{Conclusion}

Diet play important role in the management of dermatological disease such as psoriasis. Its management involves a host of factors apart from conventional therapy. A variety of complete dietary intolerance has been responsible for the psoriasis lesion and associated skin disorder. Daily routine changes and dietary changes should form an important phase of these interventions. Gluten intolerance has been increased in the population and eventually a number of researchers have been advised for gluten free diet. Still Gluten free diet assisted in improving psoriasis with no Celiac Disease but with IgA and/or IgG AGA. The alcohol, smoking dependence of patients with psoriasis has led to its worsening. Reduction in the level of Vitamin A and Vitamin B9 has been reported and therefore it can be recommended to enhance level of both the vitamins dietary intake. Similar is the case of vitamin D can be useful in the treatment of Psoriasis. Intake of fresh green leafy vegetables and fruits was associated with a lower risk of psoriasis and it can be helpful to psoriasis patients due to low Arachidonic acid (AA). The useful role of antioxidants like zinc, taurine, and selenium has also been established. Though, a condensed research focusing more on advantages and disadvantages of dietary intake is needed so that a clear relationship between diet or nutritional habits can be established with aetiology and pathogenesis of psoriasis. Dermatologists required to conscious of the different dietary management and the evidence regarding the efficacy and safety of these.

\section{Acknowledgement}

The authors are thankful to teaching and non teaching staff of Durgamata institute of pharmacy and Poona College of pharmacy.

\section{Bibliography}

1. Singh KK and Tripathy S. "Natural treatment alternative for psoriasis: a review on herbal resources". Journal of Applied Pharmaceutical Science 4.11 (2014): 114-121. 
2. Kuchekar., et al. "“Psoriasis: A comprehensive review”. International Journal of Pharmacy and Life Sciences 2.6 (2011).

3. Nasir A., et al. "Unani and modern aspects of psoriasis (Da'uus-Sadaf) treatment: a review". International Journal of Research in Dermatology 6.4 (2020): 589-596.

4. Tripathi P and Bhardwaj P. "Psoriasis: An autoimmune disorder". Journal of Drug Delivery and Therapeutics 10.5 (2020): 316-324.

5. Wolters M. "Diet and psoriasis: experimental data and clinical evidence". British Journal of Dermatology 153.4 (2005): 706714.

6. Huang., et al. "Relationships between obesity and the clinical severity of psoriasis in Taiwan". Journal of the European Academy of Dermatology and Venereology 24.9 (2010): 1035-1039.

7. Wolk., et al. "Excessive body weight and smoking associates with a high risk of onset of plaque psoriasis". Acta dermatovenereologica 89.5 (2009): 4927.

8. Gisondi., et al. "Weight loss improves the response of obese patients with moderate-to-severe chronic plaque psoriasis to low-dose cyclosporine therapy: a randomized, controlled, investigator-blinded clinical trial". The American Journal of Clinical Nutrition 88.5 (2008): 1242-1247.

9. Burg G and Geiges M. "Lepra vulgaris. History of psoriasis". Journal of the Turkish Academy of Dermatology 8.3 (2014): $1483 r 1$.

10. Jensen., et al. "Effect of weight loss on the severity of psoriasis: a randomized clinical study". JAMA Dermatology 149.7 (2013): 795-801.

11. Del Giglio., et al. "Weight reduction alone may not be sufficient to maintain disease remission in obese patients with psoriasis: a randomized, investigator-blinded study". Dermatology 224.1 (2012): 31-37.

12. Polo., et al. "Dietary patterns of patients with psoriasis at a public healthcare institution in Brazil". Anais Brasileiros de Dermatologia 95 (2020): 452-458.

13. Duarte., et al. "The management of psoriasis through diet". Psoriasis: Targets and Therapy 2 (2012): 45-53.

14. Rastmanesh R. "Psoriasis and vegetarian diets: A role for cortisol and potassium?". Medical Hypotheses 3.72 (2009): 368.
15. Rush M and Bagel J. "The Role of a Gluten-Free Diet in Psoriasis". InPsoriasis Forum. Sage CA: Los Angeles, CA: SAGE Publications 11.4 (2011): 254-258.

16. Hagforsen., et al. "Psoriasis patients with antibodies to gliadin can be improved by a gluten-free diet". British Journal of Dermatology 132 (2000): 44-51.

17. Michaëlsson., et al. "Gluten-free diet in psoriasis patients with antibodies to gliadin results in decreased expression of tissue transglutaminase and fewer Ki67+cells in the dermis". Acta Dermato-Venereologica 83 (2003): 425-429.

18. Millsop., et al. "Diet and psoriasis, part III: role of nutritional supplements". Journal of the American Academy of Dermatology 71.3 (2014): 561-569.

19. Márquez-Balbás., et al. "Study on the use of omega-3 fatty acids as a therapeutic supplement in treatment of psoriasis". Clinical, Cosmetic and Investigational Dermatology 4 (2011): 73.

20. Passi., et al. "Psoriasis and diet". Progress in Nutrition 6.4 (2004): 231-247.

21. Upala., et al. "Effect of omega-3 fatty acids on disease severity in patients with psoriasis: a systematic review". International Journal of Rheumatic Diseases 20.4 (2017): 442-450.

22. Usedom., et al. Journal of Clinical and Experimental Dermatology Research 8 (2017): 5.

23. Kanda., et al. "Nutrition and psoriasis". International Journal of Molecular Sciences 21.15 (2020): 5405.

24. Kim., et al. "Association between homocysteine levels and psoriasis: a meta-analysis". Annals of Dermatology 31.4 (2019): 378-386.

25. McDonald., et al. "A review of psoriasis, a known risk factor for cardiovascular disease and its impact on folate and homocysteine metabolism". Journal of Nutrition and Metabolism (2012).

26. Uslu., et al. "Blood homocysteine, folic acid, vitamin B12 and vitamin B6 levels in psoriasis patients". TURKDERM-Turkish Archives of Dermatology and Venereology 51.3 (2017): 92-97.

27. Lin., et al. "Homocysteine and psoriasis". Bioscience Reports 39.11 (2019): BSR20190867. 
28. Barrea., et al. "Vitamin D and its role in psoriasis: An overview of the dermatologist and nutritionist". Reviews in Endocrine and Metabolic Disorders 18.2 (2017): 195-205.

29. Perez., et al. "Safety and efficacy of oral calcitriol (1, 25-dihydroxyvitamin D3) for the treatment of psoriasis". British Journal of Dermatology 134.6 (1996): 1070-1078.

30. Fu LW and Vender R. "Systemic role for vitamin D in the treatment of psoriasis and metabolic syndrome". Dermatology Research and Practice (2011).

31. Nazıroğlu., et al. "Selenium and psoriasis". Biological Trace Element Research 150.1 (2012): 3-9.

32. Madhulatha M and Vijayabhaskar M. "Evaluation of Therapeutic Efficacy of Antioxidants in Psoriatic Cases". Indian Journal of Medical Biochemistry 23.1 (2019): 167-169.

33. Smith., et al. "Complementary and alternative medicine for psoriasis: a qualitative review of the clinical trial literature". Journal of the American Academy of Dermatology 61.5 (2009): 841-856.

34. Amin., et al. "Role of dietary intervention in psoriasis: A review". IJCD 1.1 (2018): 13.

35. Zeng., et al. "Critical role of environmental factors in the pathogenesis of psoriasis". The Journal of Dermatology 44.8 (2017): 863-872.

36. Menter., et al. "Guidelines of care for the management of psoriasis and psoriatic arthritis: Section 1. Overview of psoriasis and guidelines of care for the treatment of psoriasis with biologics". Journal of the American Academy of Dermatology 58.5 (2008): 826-850.

37. Kamiya., et al. "Risk factors for the development of psoriasis". International Journal of Molecular Sciences 20.18 (2019): 4347.

38. Temiz., et al. "The effect of smoking on the psoriasis: Is it related to nail involvement?". Dermatologic Therapy 33.6 (2020): e13960.

39. Bélanger., et al. "Plaque psoriasis: understanding risk factors of this inflammatory skin pathology". Journal of Cosmetics, Dermatological Sciences and Applications 6.2 (2016): 67.

40. Naldi L. "Psoriasis and smoking: links and risks". Psoriasis (Auckland, NZ) 6 (2016): 65.
41. Singh., et al. "Psoriasis An overview". World Journal of Pharmaceutical Sciences 3.8 (2015): 1732-1740.

42. Wu AG and Weinberg JM. "The impact of diet on psoriasis". Cutis 104.2 (2019): 7-10.

43. Choudhary., et al. "Psoriasis: Role of dietary management in diminution of its symptoms". Bioscience Biotechnology Research Communications 9 (2016): 391-398.

Volume 4 Issue 10 October 2021

(C) All rights are reserved by Abdul Haque Bamer., et al. 